NASA Technical Memorandum 107650

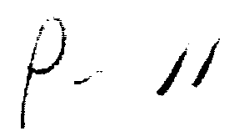

\title{
TIME SIMULATION OF FLUTTER WITH LARGE STIFFNESS CHANGES
}

Mordechay Karpel and Carol D. Wieseman

July 1992

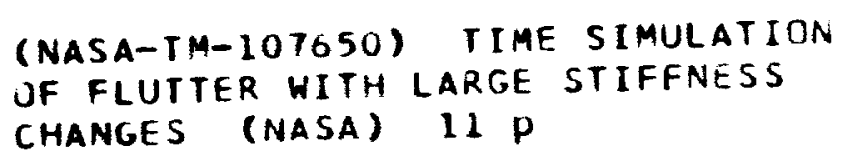

N92-32111

Unclas

$63 / 05 \quad 0116457$ 


\section{$9 \cdot$}




\title{
TIME SIMULATION OF FLUTTER WITH LARGE STIFFNESS CHANGES
}

\author{
M. Karpel* \\ Technion - Israel Institute of Technology \\ Haifa, Israel \\ C.D. Wieseman ${ }^{\dagger}$ \\ NASA Langley Research Center \\ Hampton, Virginia
}

\begin{abstract}
Time simulation of flutter, involving large local structural changes, is formulated with a state-space model that is based on a relatively small number of generalized coordinates. Free-free vibration modes are first calculated for a nominal finite-element model with relatively large fictitious masses located at the area of structural changes. A low-frequency subset of these modes is then transformed into a set of structural modal coordinates with which the entire simulation is performed. These generalized coordinates and the associated oscillatory aerodynamic force coefficient matrices are used to construct an efficient time-domain, state-space model for a basic aeroelastic case. The time simulation can then be performed by simply changing the mass, stiffness and damping coupling terms when structural changes occur. It is shown that the size of the aeroelastic model required for time simulation with large structural changes at a few apriori known locations is similar to that required for direct analysis of a single structural case. The method is applied to the simulation of an aeroelastic wind-tunnel model. The diverging oscillations are followed by the activation of a tip-ballast decoupling mechanism that stabilizes the system but may cause significant transient overshoots.
\end{abstract}

\section{Nomenclature}

[A] system matrix

$\left[A_{1}\right],\left[A_{2}\right]$ matrix coefficients of aerodynamic approximation

$b \quad$ reference semichord

[B] damping matrix of nominal structure

[C] output matrix

$[D],[E]$ matrix coefficients of aerodynamic approximation

*Associate Professor, Aerospace Engineering, Member AIAA.

Aerospace Engineer, Member AIAA.
$[G B]$

$[G K]$

$[G M]$

$[I]$

$k$

$[K]$

$m$

$[M]$

$\left[M_{f}\right]$

$n$

$n_{f}$

$q$ $[Q(i k)]$

$\left[\tilde{Q}_{b}(\bar{s})\right]$

$[R]$

$s$

$\bar{s}$

$t$

V

$\{x\}$

$\left\{x_{a}\right\}$

$\{y\}$

$[\Delta B]$

$[\Delta K]$

$[\Delta M]$

$\lambda$

[ל]

$\{\xi\}$

[ $\phi]$ matrix of vibration modes in discrete coordinates

$[\psi] \quad$ eigenvectors of $[A]$

[X] undamped eigenvectors in modal coordi-

nates

$\omega \quad$ vibration frequency

$\left[\omega_{n}\right]$ diagonal matrix of natural frequencies

$\omega_{n} \quad$ natural vibration modal frequency 


\section{Subscripts}

$\begin{array}{ll}a & \text { actual case of stiffness variation } \\ \text { accl } & \text { accelerations } \\ b & \text { basic structural modes } \\ d & \text { direct model } \\ \text { disp } & \text { displacements } \\ f & \text { fictitious masses } \\ F & \text { flutter } \\ r & \text { response locations } \\ T & \text { transition in stiffness } \\ v e l & \text { velocities }\end{array}$

\section{Abbreviations}

$\begin{array}{ll}\text { AFW } & \text { Active Flexible Wing } \\ \text { FM } & \text { ficticious mass } \\ \text { GAF } & \text { generalized aerodynamic force } \\ \text { MS } & \text { Minimum State }\end{array}$

\section{Introduction}

The common approach for formulating the equations of motion of aeroelastic systems starts with a normal modes analysis of the structural model ${ }^{1}$. Unsteady aerodynamic force coefficient matrices are then calculated at various reduced frequency values to account for the aerodynamic coupling between these modes while undergoing oscillatory motion. Classical frequencydomain aeroelastic analysis methods ${ }^{2}$ use the modal structural properties and the tabulated aerodynamic matrices to calculate the flutter conditions at which the aeroelastic system matrix is neutrally stable. The basic assumption of the modal approach is that the structural deflections of the aeroelastic system are linear combinations of a limited set of low frequency vibration modes.

Time-domain aeroelastic modeling techniques, which transform the equations of motion into a state-space, time-invariant form, call for the approximation of the aerodynamic matrices by rational functions in the Laplace domain. The order of the resulting state-space model is a function of the number of selected modes, the number of aerodynamic approximation roots, and the approximation formula. The main considerations in constructing the model are its size (which affects the efficiency of the subsequent analyses), its accuracy, and the model construction efforts. Tiffany and Adams ${ }^{3}$ summarized and extended the most commonly used aerodynamic approximation methods. Among those, the minimum-state method of Karpel ${ }^{4-6}$ yields the smallest state-space aeroelastic models per desired accuracy. Being based on an iterative nonlinear leastsquare solution, the minimum-state method requires larger model-construction computer time, but the extra time is minor relative to the time savings in typical subsequent analyses.
Various computational schemes such as structural optimization, parametric studies, the investigation of damage effects, and structural changes during dynamic response, require repeated construction of the model for numerous structural variations. Repeated calculation of the normal modes and the associated aerodynamic matrices every time the structure changes is often impractical in these cases. A more practical approach is to introduce structural changes without changing the modal coordinates. The validity of this approach depends on the structural information contained in the modal coordinates and the magnitude of the structural changes. The number of required modes increases with the magnitude of the allowable structural move limits.

Keeping the modal coordinates unchanged is even more important in time simulation of aeroelastic response during which structural changes occur. The occurrences of structural changes define the time segments between the model changes. The end conditions of one segment are the initial conditions of the following one. One can argue that these transition-point conditions can be transformed to a set of new coordinates. But this transformation is adequate only if the new coordinates can be expressed as a linear combination of the old ones. However, if this is the case, there is also no need to change the modal coordinates in the first place. An aeroelastic optimization procedure ${ }^{7}$ where a realistic wing structure was optimized with aeroservoelastic stability constraints, demonstrated that moderate structural changes can be accommodated without changing the basic 25 modal coordinates. In order to accommodate large structural changes, the modes that serve as generalized coordinates must contain significant distortions in the vicinity of the changes. Taking into account more modes supports this purpose but may result in an excessively large aeroelastic model.

A method for accommodating large structural changes at a small number of structural locations, without significantly increasing the model size has been presented in reference 8 . The procedure starts with calculating a set of low frequency vibration modes with the structure loaded with large fictitious masses at the locations of anticipated structural changes. The fictitious masses cause the vibration modes to contain the local deformations required for an adequate accommodation of large structural changes. The fictitious-mass idea has also been applied in the context of substructure modal coupling ${ }^{9,10}$ and eigenvalue sensitivities of control augmented structures ${ }^{11}$.

The purpose of this work is to outline the process of applying the fictitious-mass approach to generate efficient fixed-coordinate time-domain aeroelastic models for dynamic response with structural changes, and to demonstrate the use of these models for time simulation of flutter during which large local stiffness changes occur. 


\section{Analytical Development}

Direct Time-Domain Analysis

Time-domain, first-order, state-space formulation of aeroelastic systems starts (as in the classic secondorder, frequency-domain case) with zero-damping normal modes analysis of a finite-element model of the flight vehicle. A set of low-frequency vibration modes, $[\phi]$, is then used to calculate the generalized unsteady aerodynamic force (GAF) coefficient matrices, $[Q(i k)]$, for various reduced frequency values. Timedomain modeling requires these GAF matrices to be approximated by rational interpolation functions in the Laplace $(s)$ domain. The aerodynamic approximations in this work are performed by the Minimum-State (MS) method ${ }^{4,5}$. The approximation function, constrained to exactly match steady aerodynamics, is

$$
\begin{array}{r}
{[\tilde{Q}(\bar{s})]=[Q(0)]+\left[A_{1}\right] \bar{s}+\left[A_{2}\right] \bar{s}^{2}+} \\
{[D](\bar{s}[I]-[R])^{-1}[E] \bar{s}}
\end{array}
$$

where $\bar{s}=s b / V$. The user defines the $m \times m$ diagonal aerodynamic lag matrix $[R]$ and two additional approximation constraints (for each term) which define the $\left[A_{1}\right]$ and $\left[A_{2}\right]$ matrices as functions of the other coefficients and the tabulated data. The $[D]$ and $[E]$ real coefficient matrices are calculated by an iterative nonlinear least-square procedure that fits the tabulated data matrices. The data terms can be weighted in the least-square process according to their relative importance. The physical weighting algorithm ${ }^{5,6}$ used in this work weights the tabulated data according to openloop aeroelastic response characteristics at a selected dynamic pressure. It has been shown in various applications that the resulting model is applicable to openand closed-loop analyses with large dynamic pressure variations $s^{5,6,12,13}$.

A full description of the state-space aeroservoelastic model resulting from the MS approximation is given in reference 14 . The state-space equation of motion of the aeroelastic system without external excitation is

$$
\{\dot{x}\}=[A]\{x\}
$$

where

$$
\begin{gathered}
\{x\}=\left\{\begin{array}{c}
\xi \\
\dot{\xi} \\
x_{a}
\end{array}\right\} \\
{[A]=\left[\begin{array}{ccc}
0 & {[I]} & 0 \\
-[\bar{M}]^{-1}[\bar{K}] & -[\bar{M}]^{-1}[\bar{B}] & -q[\bar{M}]^{-1}[D] \\
0 & {[E]} & \frac{V}{b}[R]
\end{array}\right]} \\
{[\bar{M}]=[G M]+\frac{q b^{2}}{V^{2}}\left[A_{2}\right]}
\end{gathered}
$$

$$
\begin{aligned}
& {[\bar{K}]=[G K]+q[Q(0)]} \\
& {[\bar{B}]=[G B]+\frac{q b}{V}\left[A_{1}\right]}
\end{aligned}
$$

where the $[G M]$ is the diagonal generalized mass matrix found in the normal modes analysis, and $\left[G K^{*}\right]$ is the diagonal stiffness matrix whose elements are $G K_{i}=G M_{i} \omega_{n}^{2}$. When the structural damping characteristics are calculated from structural element properties (which is rarely the case), the normal modes are still calculated with zero damping, and the resulting generalized damping matrix is

$$
[G B]=[\phi]^{T}[B][\phi]
$$

which is a full matrix. In most cases, however, the generalized damping matrix is assumed to be the diagonal matrix

$$
[G B]=2[\zeta][G M]\left[\omega_{n}\right]
$$

where the diagonal matrix $[\zeta]$ is either chosen using engineering judgment (typical values are 0.005 to 0.02 ) or measured in a ground vibration test. The dynamic response at specified response locations is related to the response of the states of Eq. (2) by

$$
\{y(t)\}=[C]\{x(t)\}
$$

where $[C]$ is based on the modal deflections at the response locations, $\left[\phi_{r}\right]$. Structural displacements are calculated with

$$
\left[C_{\text {disp }}\right]=\left[\begin{array}{lll}
\phi_{r} & 0 & 0
\end{array}\right]
$$

structural velocities are calculated with

$$
\left[C_{v e l}\right]=\left[\begin{array}{lll}
0 & \phi_{r} & 0
\end{array}\right]
$$

and structural accelerations are calculated with

$$
\left[C_{a c c l}\right]=-\left[\phi_{r}\right][\bar{M}]^{-1}\left[\begin{array}{ccc}
\bar{K} & \bar{B} & q D
\end{array}\right]
$$

The state response to initial conditions can be expressed analytically as

$$
\{x(t)\}=[\psi]\left[e^{\lambda t}\right][\psi]^{-1}\{x(0)\}
$$

where $\left[e^{\lambda t}\right]$ is a diagonal matrix where the $\lambda$ values are the eigenvalues of $[A]$, and $[\psi]$ is the matrix of associated eigenvectors. Most of the eigenvalues and their associated eigenvectors are complex. Since the complex ones appear in conjugate pairs, the resulting $\{x(t)\}$ is always real, and is actually calculated by real-number algebra.

\section{Structural Changes by Direct Modal Coupling}

Structural changes that occur during the dynamic response, due to nonlinearities or application of mechanical devices, modify all or some of the finite-element $[M],[K]$ and $[B]$ matrices by the increments $[\Delta M]$, $[\Delta K]$ and $[\Delta B]$ respectively. With the assumption that 
the structural displacements after the changes can still be expressed as linear combinations of the original vibration modes, the analysis can continue with $[G M]$, $[G K]$ and $[G B]$ of $\mathrm{Eq}$. (2) replaced by

$$
\begin{gathered}
{\left[G M_{a}\right]=[G M]+[\phi]^{T}[\Delta M][\phi]} \\
{\left[G K_{a}\right]=[G K]+[\phi]^{T}[\Delta K][\phi]}
\end{gathered}
$$

and

$$
\left[G B_{a}\right]=[G B]+[\phi]^{T}[\Delta B][\phi]
$$

The physically-weighted aerodynamic approximation performed for the original model is assumed to remain valid for the modified model. This has been demonstrated in stability analysis with moderate ${ }^{7}$ and large ${ }^{8}$ structural changes.

When the structural changes occur at time $t=t_{a}$, the state response of $\mathrm{Eq}$. (9) is continued with

$$
\{x(t)\}=\left[\psi_{a}\right]\left[e^{\lambda_{a}\left(t-t_{e}\right)}\right]\left[\psi_{a}\right]^{-1}\left\{x\left(t_{a}\right)\right\}
$$

where $\lambda_{a}$ and $\left[\psi_{a}\right]$ are the eigenvalues and eigenvectors of the modified system matrix [A]. It should be noted that the modal coordinates do not change when the $[A]$ matrix varies. Consequently, the response coefficient matrix $[C]$ of Eq. (5) remains unchanged, except for that of the acceleration response, $\mathrm{Eq} . \quad(8)$, which is now based on Eqs. (10)-(12).

The flutter characteristics of the system before and after the structural changes are calculated by root-locus analyses of the respective $[A]$ matrices with variable dynamic pressure.

\section{The Fictitious-Mass Model}

The application of the direct modal coupling approach with large structural changes would require the inclusion of a large number of vibration modes. A way to analyze the system stability for large local structural variations with a minimal increase of the model size and without changing the modal coordinates was presented in reference 8 . The method is extended here to deal with time responses. The finite-element vibration modes and the associated GAF's are calculated as above for a nominal case, but with the locations of anticipated structural changes loaded with large fictitious masses. A set of $n_{f}$ low-frequency fictitious vibration modes, $\left[\phi_{f}\right]$, is selected for further analysis. A coordinate transformation is then performed to "clean out" the fictitious masses and to form an actual "basic" case whose mass and stiffness matrices may differ from those of the nominal case by $\left[\Delta M_{b}\right]$ and $\left[\Delta K_{b}\right]$. The transformation is based on the natural frequencies $\left[\omega_{b}\right]$ and eigenvectors $\left[\chi_{b}\right]$ (normalized to unit generalized masses) associated with the equation of free undamped vibrations in modal coordinates

$$
\begin{array}{r}
\left(\left[G M_{f}\right]+\left[\phi_{f}\right]^{T}\left[\Delta M_{b}-M_{f}\right]\left[\phi_{f}\right]\right)\left\{\ddot{\xi}_{f}\right\}+ \\
\left(\left[G K_{f}\right]+\left[\phi_{f}\right]^{T}\left[\Delta K_{b}\right]\left[\phi_{f}\right]\right)\left\{\xi_{f}\right\}=\{0\}
\end{array}
$$

The mode shapes and GAF's calculated for the fictitious-mass (FM) finite-element model are transformed to the basic case by

$$
\left[\phi_{b}\right]=\left[\phi_{f}\right]\left[\chi_{b}\right]
$$

and

$$
\left[Q_{b}(i k)\right]=\left[\chi_{b}\right]^{T}\left[Q_{f}(i k)\right]\left[\chi_{b}\right]
$$

The basic-case mode shapes $\left[\phi_{b}\right]$ serve as a constant set of structural generalized coordinates throughout the response analysis (as was $[\phi]$ in the direct modal approach). When the columns of $\left[\phi_{b}\right]$ and the associated frequencies $\left[\omega_{b}\right]$ are compared to those calculated directly for the basic-case finite-element model, they appear in two groups. The low-frequency group of $n_{d}$ modes is practically identical to the directly calculated modes. The $n_{f}-n_{d}$ modes in the high-frequency group, whose number and nature depend on the number and magnitudes of the fictitious masses, do not represent actual normal modes, but are synthetic modes with relatively large local distortions in the vicinity of the fictitious masses. In this way, the modes contain detailed local structural information with a minimal increase of the number of modes.

The MS approximation of Eq. (1) is now calculated with the transformed modal data, and the statespace Eq. (2) and the acceleration response matrix (8) are constructed for the basic case with $[G M]=[I]$, $[G K]=\left[\omega_{b}\right]^{2}$ and $[G B]=2\left[\zeta_{b}\right]\left[\omega_{b}\right]$ (in the case of diagonal generalized damping). When the structural damping characteristics are calculated from structural element properties, $\left[G B_{f}\right]$ is first calculated from the FM finite-element model using Eq. (3) with $[\phi]=\left[\phi_{f}\right]$, and then transformed to the basic case in the same way the GAF matrices are transformed in Eq. (16).

Most of the response analysis from this point on is performed in the way shown above for the direct modal approach, Eqs. (9)-(13), with $[\phi]=\left[\phi_{b}\right]$. An exception is the generalized damping matrix, $\left[G B_{a}\right]$ of $\mathrm{Eq}$. (12), when large mass and/or stiffness changes appear in Eqs. (10) and (11). With no damping changes, $\left[G B_{a}\right]$ would remain diagonal but would cause, in effect, damping coupling between the normal modes of the modified system $^{8}$. In order to yield an effectively diagonal structural damping matrix, $[G B]$ is replaced by

$$
\left[G B_{a}\right]=2\left[G M_{a}\right]\left[\chi_{a}\right]\left[\zeta_{a}\right]\left[\omega_{a}\right]\left[\chi_{a}\right]^{T}\left[G M_{a}\right]
$$

where $\left[\omega_{a}\right]$ and $\left[\chi_{a}\right]$ are the complete set of frequencies and eigenvectors associated with

$$
\left[G M_{a}\right]\left\{\ddot{\xi}_{b}\right\}+\left[G K_{a}\right]\left\{\xi_{b}\right\}=\{0\}
$$

where $\left[\chi_{a}\right]$ is normalized to yield

$$
\left[\chi_{a}\right]^{T}\left[G M_{a}\right]\left[\chi_{a}\right]=[I]
$$

The accuracy of the FM aeroelastic model (which is based on $n_{f}$ modes) can be evaluated by separate com- 
parisons of time responses of several structural variations to those obtained directly with their own $n_{d}$ natural modes. Since the FM models contain more structural information, the structural initial conditions have to be defined first in terms of the coordinates of the direct models. With $\left\{\xi_{d}(0)\right\}$ and $\left\{\xi_{d}(0)\right\}$ being the directmodel displacement and velocity initial conditions of a comparison case, the equivalent initial conditions in the associated FM case are

$$
\left\{\xi_{f}(0)\right\}=\left[\bar{\chi}_{a}\right]\left\{\xi_{d}(0)\right\}
$$

and

$$
\left\{\dot{\xi}_{f}(0)\right\}=\left[\bar{x}_{a}\right]\left\{\dot{\xi}_{d}(0)\right\}
$$

where the columns of the $n_{f} \times n_{d}$ transformation matrix $\left[\bar{\chi}_{a}\right]$ are the $n_{d}$ lowest-frequency eigenvectors associated with free FM modal equation of motion (18) for this case.

\section{Results}

The numerical example consists of a mathematical model of the Active Flexible Wing (AFW) wind-tunnel model ${ }^{15,16}$ tested at the NASA Langley Research Center. An external ballast is connected to the tip of the wing through a mechanism designed to decouple the pitch motion of the ballast from that of the wing when flutter occurs. Figure 1 shows a close-up view of the ballast and its attachment to the wing. Details of the structural and aerodynamic models are given in reference 8 . A frequency-domain flutter analysis by the $p-k$ method $^{2}$ showed that, with "stiff" or "coupled" pitch connection, the Mach 0.9 antisymmetric flutter dynamic pressure is about 1.9 psi and the flutter frequency is about $12 \mathrm{~Hz}$. When the decoupling mechanism is activated, the pitch connection stiffness is reduced by $96.5 \%$ ("soft" or "decoupled") and the flutter dynamic pressure changes to about 3.0 psi and the flutter frequency to $31 \mathrm{~Hz}$. The decoupling device thus provides a drastic change in both flutter dynamic pressure and the flutter mechanism.

Three time-domain aeroelastic models, starting from separate NASTRAN normal modes analyses, were constructed. Two were direct "coupled" and "decoupled" models and the third was an FM model. The generalized aerodynamic force coefficient matrices in each case were calculated at 14 reduced frequency values between 0 and 1.5. Minimum-State, physically weighted rational function approximations of the unsteady aerodynamics ${ }^{5}$ were performed with 8 lag terms, which yielded 8 aerodynamic augmenting states. The physical weightings were performed at $q=1.5 \mathrm{psi}$. Each model had two versions, one where the approximation was constrained to match the real and imaginary parts of the aerodynamic data at $k=1.5$, and one with the real data matching constraint replaced by $\left[A_{2}\right]=0$ in Eq. (1), to avoid repetitive inversions of $[\bar{M}]$ in Eq. (2). All the models were constructed with diagonal modal damping values of $\zeta=0.01$.
The FM NASTRAN model was with the "decoupled" pitch connection and with a fictitious pitch inertia of $3 \mathrm{lb}$-in- $\sec ^{2}$, twice that of the tip ballast, loading the wing end of the connection pitch spring. A set of 14 low frequency fictitious-mass modes, $\left[\phi_{f}\right]$, was used to generate the basic "coupled" model by solving for the natural frequencies and eigenvectors of Eq. (14), with $\left[\Delta M_{b}\right]=0$ in our case, then transforming the data to the "coupled" modal coordinates, Eqs. (15) and (16), and then performing the aerodynamic approximation and the model construction. A comparison between the first seven natural frequencies of the "coupled" model obtained directly from NASTRAN, those calculated from the 14 fictitious-mass modes and those calculated by direct coupling from 14 "decoupled" modes without fictitious masses is given in Table 1 . It is clear that the FM model produces accurate frequencies. The fluttercritical ballast pitch frequency obtained from the "decoupled" model without fictitious mass is $18 \%$ higher than the correct one, which indicates that the direct coupling approach is not adequate in our case.

The basic FM aeroelastic model was used for various stability and time-response analyses with different pitch connection stiffnesses between "coupled" and "decoupled" conditions. The transition from the basic "coupled" case to another case is performed by simply introducing the appropriate stiffness and damping coupling terms. A comparison between flutter conditions calculated by the frequency domain $p-k$ method and those obtained from root-locus analyses of the statespace models is given in Table 2 . The largest deviation of state-space from $p-k$ results is in the flutter dynamic pressure of the "decoupled" FM model with $\left[A_{2}\right]=0$. The contributors to this error are the large difference between the flutter dynamic pressure (3.102 psi) and the physical weighting dynamic pressure (1.5 psi), the large deviation from the weighting "coupled" pitch spring, and the $\left[A_{2}\right]=0$ constraint. The error however is still below $5 \%$.

A root-loci plot of the low-frequency eigenvalues of the system matrix $[A]$ where the pitch connection stiffness is varied in small increments from "coupled" to "decoupled" values at $q=2$ psi is shown in Fig. 2 . This was accomplished by varying $[A]$ from "coupled" to "decoupled" linearly. The linear variations are valid because $[\bar{M}]$ in Eqs. (2) and (8) is not affected by the changes. It should be mentioned that with linear variations, while the extreme points modal dampings produced by the application of Eq. (17) are effectively diagonal, those at intermediate points are not. However, without having specific knowledge on the damping behavior of the decoupling mechanism at intermediate points, the linear change is as good as other possible variations. The variation of the maximal real part of the eigenvalues of Fig. 2 is shown in Fig. 3. It can be observed that the system stability is very sensitive to the connection spring, especially when it is close to 


\begin{tabular}{|c|c|c|c|c|c|}
\hline \multicolumn{4}{|c|}{ REPORT DOCUMENTATION PAGE } & \multicolumn{2}{|r|}{$\begin{array}{l}\text { Form Approved } \\
\text { OMB No. 0704-0188 }\end{array}$} \\
\hline \multicolumn{6}{|c|}{ 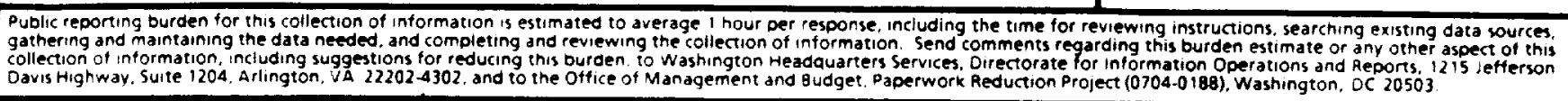 } \\
\hline \multicolumn{2}{|c|}{ 1. AGENCY USE ONLY (Leave blank) } & $\begin{array}{l}\text { 2. REPORT DATE } \\
\text { July } 1992 \\
\end{array}$ & \multicolumn{3}{|c|}{$\begin{array}{l}\text { 3. REPORT TYPE AND DATES COVERED } \\
\text { Technical Memorandum }\end{array}$} \\
\hline \multicolumn{4}{|c|}{$\begin{array}{l}\text { 4. TITLE AND SUBTITLE } \\
\text { Time Simulation of Flutter With Large Stiffness Changes }\end{array}$} & \multicolumn{2}{|c|}{$\begin{array}{l}\text { 5. FUNDING NUMBERS } \\
\text { WU 505-63-50-15 }\end{array}$} \\
\hline \multicolumn{6}{|l|}{$\begin{array}{l}\text { M. Karpel } \\
\text { C. D. Wieseman }\end{array}$} \\
\hline \multicolumn{4}{|c|}{$\begin{array}{l}\text { NASA Langley Research Center } \\
\text { Hampton, VA } 23665-5225\end{array}$} & \multicolumn{2}{|c|}{$\begin{array}{l}\text { 8. PERFORMING ORGANIZATION } \\
\text { REPORT NUMBER }\end{array}$} \\
\hline \multicolumn{4}{|c|}{ 9. SPONSORING / MONITORING AGENCY NAME(S) AND ADDAESS(ES) } & \multicolumn{2}{|c|}{$\begin{array}{l}\text { 10. SPONSORING /MONITORING } \\
\text { AGENCY REPORT NUMBER }\end{array}$} \\
\hline \multicolumn{4}{|c|}{$\begin{array}{l}\text { National Aeronautics and Space Administration } \\
\text { Washington, DC 20546-0001 }\end{array}$} & \multicolumn{2}{|c|}{ NASA TM-107650 } \\
\hline \multicolumn{6}{|c|}{$\begin{array}{l}\text { 11. SUPPLEMENTARY NOTES } \\
\text { Presented at the AIAA Structures, Structural Dynamics, and Materials Conference, Dallas, Texas, } \\
\text { April 13-15, 1992. Karpel: Technion-Israel Institute of Technology; Wieseman: NASA Langley } \\
\text { Research Center, Hampton, VA. }\end{array}$} \\
\hline \multicolumn{4}{|c|}{$\begin{array}{l}\text { 12a. DISTRIBUTION/AVAILABILITY STATEMENT } \\
\text { Unclassified - Unlimited } \\
\text { Subject Category } 05\end{array}$} & \multicolumn{2}{|c|}{ 12b. DISTRIBUTION CODE } \\
\hline \multicolumn{6}{|c|}{$\begin{array}{l}\text { 13. ABSTRACT (Maximum 200 words) } \\
\text { Time simulation of flutter, involving large local structural changes, is formulated with a state-space } \\
\text { model that is based on a relatively small number of generalized coordinates. Free-free vibration modes } \\
\text { are first calculated for a nominal finite-element model with relatively large fictitious masses located at } \\
\text { the area of structural changes. A low-frequency subset of these modes is then transformed into a set of } \\
\text { structural modal coordinates with which the entire simulation is performed. These generalized } \\
\text { coordinates and the associated oscillatory aerodynamic force coefficient matrices are used to construct } \\
\text { an efficient time-domain, state-space model for a basic aeroelastic case. The time simulation can then be } \\
\text { performed by simply changing the mass, stiffness, and damping coupling terms when structural } \\
\text { changes occur. It is shown that the size of the aeroelastic model required for time simulation with large } \\
\text { structural changes at a few apriori known locations is similar to that required for direct analysis of a } \\
\text { single structural case. The method is applied to the simulation of an aeroelastic wind-tunnel model. } \\
\text { The diverging oscillations are followed by the activation of a tip-ballast decoupling mechanism that } \\
\text { stabilizes the system but may cause significant transient overshoots. }\end{array}$} \\
\hline \multirow{2}{*}{\multicolumn{5}{|c|}{$\begin{array}{l}\text { 14. SUBJECT TERMS } \\
\text { Direct modal coupling; fictitious mass; flutter; generalized coordinates; } \\
\text { large stiffness changes; minimum-state aerodynamics; reduced order modeling; } \\
\text { time simulation }\end{array}$}} & $\begin{array}{l}\text { 15. NUMBER OF PAGES } \\
10\end{array}$ \\
\hline & & & & & $\begin{array}{l}\text { 16. PAICE CODE } \\
A 02\end{array}$ \\
\hline $\begin{array}{l}\text { 17. SECURITY CLASSIFICATION } \\
\text { OF REPORT } \\
\text { Unclassified }\end{array}$ & & $\begin{array}{l}\text { ECUAITY CLASSIFICATION } \\
\text { F THIS PAGE } \\
\text { classified }\end{array}$ & 19. SECI & ATION & 20. LIMITATION OF ABSTRACT \\
\hline
\end{tabular}

